\title{
CAN THE Mind WANDER INTENTIONALLY?
}

Samuel Murray, Duke University

Kristina Krasich, Duke University

Forthcoming in Mind \& Language

\begin{abstract}
Mind wandering is typically operationalized as task-unrelated thought. Some argue for the need to distinguish between unintentional and intentional mind wandering, where an agent voluntarily shifts attention from task-related to task-unrelated thoughts. We reveal an inconsistency between the standard, task-unrelated thought definition of mind wandering and the occurrence of intentional mind wandering (together with plausible assumptions about tasks and intentions). This suggests that either the standard definition of mind wandering should be rejected or that intentional mind wandering is an incoherent category. Solving this puzzle is critical for advancing theoretical frameworks of mind wandering.
\end{abstract}

Keywords: Mind Wandering; Intentional Action; Cognitive Psychology; Task-Unrelated Thought

\section{Introduction}

There has been a surge of interest in mind wandering in psychology (see Smallwood \& Schooler, 2015 for a review) and, more recently, philosophy (Dorsch, 2015; Irving, 2016; Metzinger, 2013; Sripada, 2018). Mind wandering is typically defined as task-unrelated thought in psychological research. ${ }^{1}$ More precisely, an agent mind wanders when and

\footnotetext{
${ }^{1}$ Mills et al., 2018 found that over $90 \%$ of papers published on mind wandering in 2016 assumed (implicitly or explicitly) the task-unrelated thought definition of mind wandering (Mills and colleagues do not, however, endorse the task-unrelated thought conception of mind wandering in their survey). Seli and colleagues confirm this in their recent theoretical piece on mind wandering: "To date, the most common definitions of mind-wandering include task-unrelated thought.... and stimulus-indpendent task-unrelated thought" (Seli et al., 2018-b: 479-80). Additionally, one of the earliest theoretical discussions of mind wandering proposed something close to a task-unrelated thought conception of mind wandering (Smallwood \& Schooler, 2006; cf. Giambra, 1995). This same conception was reiterated in a later review piece summarizing ten years of work on mind wandering (Smallwood \& Schooler, 2015).
} 
only when that agent has a task-unrelated, stimulus independent thought. We will refer to this as the Standard View. ${ }^{2}$

Some have recently argued for a distinction between unintentional and intentional mind wandering, where some agent either involuntarily shifts attention to task-unrelated thoughts or, instead, can engage higher order, deliberate control and choose to shift attention (e.g., Seli, Risko, \& Smilek, 2016; Seli, Risko, Smilek, \& Schacter, 2016; Irving, Manuscript). The basis of the distinction is that these two kinds of mind wandering seem to independently predict variables of interest (Seli, Risko, \& Smilek, 2016). This is potentially significant for the empirical study of mind wandering, as models of mind wandering would have to accommodate the occurrence of intentional mind wandering.

This is also potentially significant for philosophical views of mind wandering and certain philosophical conceptions of action. Recently, some have suggested that mind wandering is inherently passive despite possessing some characteristics of activity (Irving, 2016: 549-50). Because mind wandering is inherently passive, if there is such a thing as intentional mind wandering, then it would seem to be a prime example of mental behavior that lies somewhere between pure passivity and pure activity.

We argue that things are not so simple. We generate a contradiction from the combination of the Standard View and the fact that intentional mind wandering occurs (together with some innocent assumptions about tasks and intentions). This shows that either the Standard View should be rejected or that intentional mind wandering is an incoherent category.

\footnotetext{
${ }^{2}$ The Standard View is not so standard among philosophers. Philosophical inquiry into mind wandering is relatively young, however, so we do not think there is any standard view on the matter among philosophers.
} 


\section{The Puzzle}

We argue in this section that the construct of intentional mind wandering is inconsistent with the Standard View. We call this the Puzzle of Willful Wandering (PWW).

Informally, PWW shows that an agent cannot intend to have task-unrelated thoughts, as intending to have any thought thereby makes that thought related to one's task. Thus, the thoughts cannot be task-unrelated and so cannot constitute mind wandering in the standard sense:

\begin{tabular}{|c|c|c|}
\hline PWW-1 & $\begin{array}{l}\mathrm{S} \text { is mind wandering when and only when } \mathrm{S} \text { has at least one task- } \\
\text { unrelated thought }\end{array}$ & Premise \\
\hline PWW-2 & $\mathrm{S}$ is able to intentionally mind wander & Supposition \\
\hline PWW-3 & $\begin{array}{l}\text { Intentionally A-ing requires either a proximal intention to } \mathrm{A} \text { or some } \\
\text { intention to } \mathrm{B} \text { where } \mathrm{A} \text {-ing is within the motivational potential to } \mathrm{B}\end{array}$ & Premise \\
\hline PWW-4 & $\begin{array}{l}\text { Some activity is within the motivational potential of an intention to B } \\
\text { only if that activity is a constitutive means of B-ing or is an expected } \\
\text { side effect of B-ing. }\end{array}$ & Premise \\
\hline PWW-5 & $\begin{array}{l}\text { Hence, } \mathrm{S} \text { has either a proximal intention to mind wander or an } \\
\text { intention to B where mind wandering is a constitutive means of B-ing } \\
\text { or is an expected side effect of B-ing. }\end{array}$ & $2,3,4$ \\
\hline PWW-6 & If S proximally intends to A, then A-ing becomes S's task. & Pr \\
\hline PWW-7 & $\begin{array}{l}\text { If S proximally intends to mind wander, then mind wandering } \\
\text { becomes S's task. }\end{array}$ & $\begin{array}{l}5, \text { Instance } \\
\text { of } 6\end{array}$ \\
\hline PWW-8 & $\begin{array}{l}\text { An agent's thought is task-related when and only when that thought } \\
\text { contributes to her task performance. }\end{array}$ & \\
\hline PWW-9 & $\begin{array}{l}\text { Trivially, thoughts during mind wandering contribute to mind } \\
\text { wandering. }\end{array}$ & Pr \\
\hline PWW-10 & $\begin{array}{l}\text { Hence, if S proximally intends to mind wander, then S's mind } \\
\text { wandering is task-related. }\end{array}$ & 6, \\
\hline PWW-11 & Therefore, S does not proximally intend to mind wander. & $1,5,10$ \\
\hline PWW-12 & $\begin{array}{l}\text { If S's mind wandering is a constitutive means of her B-ing, then her } \\
\text { task-unrelated thought is necessary for her B-ing. }\end{array}$ & Premise \\
\hline PWW-13 & A thought unrelated to B-ing is not necessary for B-ing. & \\
\hline PWW-14 & Hence, S's mind wandering is not a constitutive means of her B-ing. & $1,5,12,13$ \\
\hline PWW-15 & $\begin{array}{l}\text { If S's mind wandering is an expected side effect of her B-ing, then } \mathrm{S} \\
\text { must be able, on some occasion, to proximally intend to mind wander. }\end{array}$ & Premise \\
\hline PWW-16 & S's mind wanderin & \\
\hline PWW-17 & Contradiction & $5,11,14,16$ \\
\hline
\end{tabular}


In the following sections, we consider some flawed responses to the puzzle and suggest that either PWW-1 or PWW-2 is false.

\section{Intentionality and Motivational Potential (PWW-3)}

Consider PWW-3, which makes a claim about the nature of intentionality in action. This claim is typically considered to be part of the Planning Theory of Intention, first proposed and systematically developed by Michael Bratman $(1984,1987)$. According to the Planning Theory, there are two ways for behaviors to be intentional actions. To see this, consider an example of a person moving their foot. On the Planning Theory, one way for the foot motion to be intentional is for the agent to proximally intend to move their foot (and that proximal intention causes the foot to move in the right sort of way). The Planning Theory, however, also allows for intentional actions in the absence of any such tight connection between behavior and intentions. So, suppose that moving the foot is just one step in walking across campus on the way home. In this case, the agent does not (or need not) intend to move their foot, yet, moving the foot is part of executing the larger (intended) action of getting home. This is what makes moving the foot intentional. Moving the foot inherits intentionality in virtue of being a component in this larger intended plan.

More formally, the Planning Theory of Intention states that an agent can intentionally A without intending to A only if the agent intends to B, and her A-ing falls within the motivational potential of the agent's intention to B (cf. Bratman, 1987: 21617). The notion of motivational potential is central to Bratman's distinction, but Bratman 
does not provide a precise characterization of this notion. ${ }^{3}$ He suggests that an agent's Aing is within the motivational potential of an intention to B only if the agent's executing her intention to B causes her to engage in A-ing and the agent believes that her A-ing contributes to executing her intention to B (Bratman, 1987: 123). The thoughts or behaviors that fall within the motivational potential of an intention are expected side effects of executing the intention or the constitutive means of executing an intention (Bratman, 1984: 399-401).

One reason to use the Planning Theory of Intention in generating PWW is that this theory of intentionality in action is weaker than other accounts that count some behavior as intentional only if an agent acquires an intention to perform that behavior (cf. Amaya, 2018). If PWW works with a relatively weak theory of intentionality in action, we assume that stronger theories will also generate PWW. Additionally, replacing the Planning Theory with anything weaker risks adopting an implausibly weak theory of intentionality in action that might overgeneralize the scope of intentional action. ${ }^{4}$ For this reason, we think that rejecting PWW-3 is unlikely to furnish a reasonable solution to PWW. ${ }^{5}$

\footnotetext{
${ }^{3}$ Bratman himself acknowledges this: "The notion of motivational potential is intended to mark the fact that my intention to $B$ may issue in my intentionally $A$-ing, rather than to explain it. It is a theoretical placeholder: it allows us to retain theoretical room for a more complex account of the relation between intention and intentional action while leaving unsettle the details of such an account" (emphasis original; 1987: 120).

${ }^{4}$ Another consideration that speaks in favor of the Planning Theory of Intention is the widespread influence of the theory. Bratman's planning theory has been widely adopted and has exerted influence in cognitive science, clinical domains, artificial intelligence, primatology and other fields (Yaffe \& Vargas, 2014 provide an overview of the influence of the Planning Theory of Intention). Hence, rejecting PWW-3 comes at the price of rejecting a view that has cross-disciplinary appeal. While this is not a decisive consideration, it does provide further reason to think that an alternative theory of intentionality in action is obviously preferable to targeting other premises of PWW.

${ }^{5}$ Some have recently challenged PWW-3 on the grounds that there can be intentional action without prior intentions (Herdova, 2018). This challenge focuses on the possibility of snap decisions, spontaneous actions, or habitual actions which are intentional without being triggered by a proximal intention. While the challenge is too complicated to address in full, we are skeptical that the cases used to formulate the
} 


\section{Side Effects (PWW-15)}

PWW-15 states that an expected side effect, E, of intending to B is intentional only if it is the case that an agent could proximally intend to E. The motivation for the principle stems from wanting intentionality to distribute out in ways that map onto an agent's foresight. For example, suppose you are on a sinking ship and you intentionally take the last lifeboat, knowing full well that this will leave others onboard stranded. In this case, it seems intuitive that you have also intentionally killed the people on the ship. When an agent intends to take a certain course of action - and foresees that there are certain consequences of taking that course of action — then some of those foreseen consequences inherit intentionality from the intention to take the course of action (see Aune, 1966).

We do not, however, want intentionality to distribute too pervasively into foreseen consequences. Hence, we need some principle to determine when foreseen consequences inherit intentionality and when they do not. PWW-15 states one such principle. PWW-15 is plausible because it explains some of our intuitions about the distribution of intentionality in action. You can intentionally rest your head on a pillow, but that does not make falling asleep intentional. ${ }^{6} \mathrm{PWW}-15$ explains why this is the case. Because one cannot proximally intend to fall asleep, falling asleep is not the right kind of activity to inherit intentionality from prior intentions.

challenge occur without proximal intentions. For example, consider the case of beginning to whistle a tune on a whim. In this case, perhaps having a song stuck in one's head, combined with beliefs about the context and relevant feelings, might contribute to acquiring an intention (where acquiring an intention does not require any decision). A full discussion of the challenge and a response can be found in Mele, Forthcoming. ${ }^{6}$ See Mele, 2009: 18-19. 
PWW-15 also resolves some action theoretic dilemmas. Consider Butler's (1978:

113) statement of Analysis "Problem" No. 16:

If Brown in an ordinary game of dice hopes to throw a six and does so, we do not say that he threw the six intentionally. On the other hand if Brown puts one live cartridge into a six-chambered revolver, spins the chamber as he aims it at Smith and pulls the trigger hoping to kill Smith, we would say if he succeeded that he had killed Smith intentionally. How can this be so, since in both cases the probability of the desired result is the same?

According to PWW-15, Brown does not throw a six intentionally because the throwing of a six is not an action that Brown can proximally intend to perform. Brown's killing of Smith, however, does inherit intentionality from Brown's intending to shoot Smith because the killing of Smith is something that Brown can proximally intend to perform. ${ }^{7}$

PWW-15, then, is a plausible principle that explains why some of the consequences or side effects of intentional action fail to inherit intentionality. If, however, the argument from PWW-1 to -11 is correct, then mind wandering is not the right sort of activity to inherit intentionality. Thus, solving PWW by denying PWW-15 either requires some solution to earlier parts of the puzzle or some new principle that states the conditions under which intentionality distributes out to side effects.

\section{Intentional Wandering?}

We think that rejecting any of the premises discussed thus far produces an unattractive solution to PWW. The puzzle thus exploits the apparent paradox of intentional wandering, or aiming to do some activity that is inherently aimless. ${ }^{8}$ This suggests that

\footnotetext{
${ }^{7}$ This echoes both Ross (1978) and Kraemer's (1978) solutions to Analysis Problem No. 16, both of whom appeal to differences of control to explain the asymmetry between dice-rolling and shooting. PWW-15, then, might be read as offering an interpretation of what control amounts to in this kind of situation. ${ }^{8}$ Consider, e.g., Irving (2016: 549-50): "It seems essential that mind-wandering lacks purpose; almost by definition, it contrasts with goal-directed forms of cognition like planning a trip or solving a crossword.
} 
the problematic premise will be one of those that discuss the nature of intentionality and wandering (namely, PWW-1 and -2).

What our puzzle shows is that either the Standard View is incorrect or that intentional mind wandering is an incoherent category. The issue is that the Standard View defines mind wandering partly in terms of task-relatedness. The argument thus far shows that intentionally $A$-ing requires an agent to have $A$-related thoughts (this, we take it, is the upshot of the argument from PWW-6 to PWW-10). Thus, the Standard View rules out the possibility of intentional mind wandering.

We believe that there are two potential responses to PWW. One is to reject the Standard View, while the other is to reject the possibility of intentional mind wandering. We do not want to weigh decisively in favor of one response; instead, we want to explore the implications of each response, leaving aside full consideration of either possibility for another occasion.

\subsection{Rejecting the Standard View}

One response to PWW is to reject the Standard View (PWW-1), which would add to a growing list of objections to the Standard View (see Andrews-Hanna et al. 2018; Christoff et al., 2016; Irving, 2016; Irving \& Thompson, 2018; Seli et al., 2018-b; Sripada, 2018). Alternative theories of mind wandering, such as the Family Resemblances view or the Dynamic view (discussed below), would also enjoy a theoretical advantage over their Standard View counterparts. So, PWW presents additional ammunition for those who object to the Standard View, but it also has

Consider the term 'mind-wandering' itself. Wandering is purposeless movement...To say that someone's mind is wandering, then, implies that her thinking is purposeless; it is not developing toward a goal or endpoint." 
interesting implications for both the Family Resemblances view and the Dynamic view, both of which we discuss next.

The Family Resemblances view claims that mind wandering is a heterogeneous construct with multiple, overlapping attributes that no single instance of mind wandering fully exemplifies (Seli et al., 2018-b: 482-84). Therefore, this view rejects the idea that there is any suitable necessary-and-sufficient-condition definition of mind wandering (see Seli et al., 2018-b: 482). Prototypical instances of mind wandering exhibit taskunrelatedness, but not all instances share this property. For this reason, the Family Resemblances view conflicts with the Standard View. The Family Resemblances view accepts that some instances of mind wandering might not be task-unrelated, which the Standard View takes to be a necessary feature of mind wandering. Further, the Family Resemblances view claims that different features of mind wandering are orthogonal, such as task-unrelatedness and intentionality (see Seli et al., 2018-b: 483). Hence, those working within the Family Resemblances framework sometimes talk about intentional task-unrelated thought (see, e.g., Martel et al., In Preparation). PWW shows, however, that these features are not orthogonal, as the task-unrelated feature of mind wandering is incompatible with the intentionality feature. Thus, PWW shows that intentional taskunrelated thought is impossible, which we take to be an interesting implication for the Family Resemblances view.

The Dynamic view of mind wandering defines mind wandering in terms of the relations between mental states rather than relations between some mental state and a task. Thus, Dynamic theories of mind wandering characterize it as unguided thought (Irving, 2016) or as relatively unconstrained thought (Christoff et al., 2016). These 
theories can actually explain how intentional mind wandering is possible. For example, Irving (Manuscript) argues that intentional mind wandering consists in thinking that is not guided toward any particular goal. However, individuals retain meta-control over episodes of intentional mind wandering. As Irving describes it, one exercises metacontrol over intentional mind wandering when: “...one actively initiates or maintains an unguided mode of thought, while exerting no control over where your attention is directed" (Manuscript: 13).

PWW tells us something important about this view of intentional mind wandering. As we argued in Section 3, proximally intending to mind wander is impossible, where an agent's proximally intending to $A$ issues immediately in her undertaking to $A$. Thus, intentional mind wandering cannot reflect intentionally initiating a mind wandering sequence; rather, intentional mind wandering must reflect intentionally maintaining one's mind wandering (see Irving, Manuscript). ${ }^{9}$ This suggests an interesting possibility. People cannot intentionally begin to mind wander, though they can intentionally maintain themselves in a state of mind wandering.

We think that there is much more to say here. We raise these issues merely to illustrate the utility of PWW beyond demonstrating an incompatibility between the Standard View and the possibility of intentional mind wandering. Even those who already reject the Standard View can take something interesting away from PWW.

\subsection{Rejecting Intentional Mind Wandering}

\footnotetext{
${ }^{9}$ A suggestive, though not decisive, argument for this claim can be made. Any activity that one can intentionally initiate is such that the agent could, on command, immediately undertake to perform that activity. No one, however, can mind wander on command (it is possible to put oneself in a position where mind wandering is likely, but this does not amount to following a command to mind wander immediately). So, mind wandering is not among those activities that one can intentionally initiate.
} 
Another potential response to $\mathrm{PWW}$ is to reject the possibility of intentional mind wandering (PWW-2). This might seem implausible, as there is substantial, and growing, evidence for intentional mind wandering. However, we sketch out some reasons for reframing this evidence to support an alternative (weaker) hypothesis consistent with rejecting the possibility of intentional mind wandering. Roughly, we believe that the evidence suggests the need for distinguishing mind wandering from related cognitive activity that superficially resemble mind wandering. These related activities might be focused daydreaming, motivated task-switching, personal goal processing, or something else. Importantly, none of these alternative activities are mind wandering. We also suggest a possible error theory to explain away self-reports of intentional mind wandering. What we propose is an outline of a larger case that could be made to explaining the evidence for intentional mind wandering within the confines of the Standard View.

Some researchers have found different associations of trait- and state-level variables between unintentional and intentional mind wandering and, thus, argue for the theoretical usefulness of distinguishing between the two. For instance, Seli, Risko, \& Smilek (2016) asked participants to complete either a difficult or easy version of the Sustained Attention to Response Task, a task that requires participants to respond to frequently presented items and withhold a response for infrequently presented target items. Mind wandering was measured with randomly distributed thought probes that prompted participants to report whether they were unintentionally or intentionally mind wandering at a given moment. Results showed that when collapsing across the two types of mind wandering, there was no significant difference in the overall rates of mind 
wandering between difficult and easy conditions. However, when analyzing intentional and unintentional mind wandering separately, the authors found: (1) higher rates of reported unintentional mind wandering than intentional mind wandering; (2) higher rates of reported intentional mind wandering in the easy-SART condition relative to the difficult-SART condition, and; (3) significantly higher proportion of reported unintentional mind wandering in the difficult-SART condition relative to the easy-SART condition. One significant conclusion that Seli and colleagues draw from this is: “...had we ignored the distinction between intentional and unintentional mind wandering, we would have drawn the incorrect conclusion that mind wandering remains unchanged across conditions" (Seli, Risko, \& Smilek, 2016: 688).

This data, however, is consistent with a weaker conclusion: in studies of mind wandering researchers must distinguish mind wandering from other, intentional mental activities that share certain superficial properties of mind wandering. Seli and colleagues show that participants sometimes deliberately distract themselves during a task, whereas other times they mind wander. And researchers should be careful not to lump mind wandering and these other deliberate forms of distraction together in their analyses. The decision to label this deliberate form of distraction intentional mind wandering is a theoretical choice that Seli and colleagues impose on the data.

What might this other form mental activity be? We suspect that there is no single answer to this question. But a few examples reveal how one might explain purported instances of intentional mind wandering in terms of other well-defined psychological constructs (which constructs, we should note, do not themselves include or overlap with mind wandering). This provides the proponent of the Standard View with a template for 
eliminating intentional mind wandering while accepting the work done under the banner of 'intentional mind wandering'.

For example, consider a case where an individual chooses to think about the vacation she's taking next month rather than the hedges she's currently trimming. This might seem like an instance of intentional mind wandering. But an alternative description is available. This could be an instance of focused daydreaming. However, focused daydreaming is not a kind of mind wandering (see Dorsch, 2015 for an extended argument for distinguishing focused daydreaming and mind wandering). Moreover, research shows that intentional mind wandering correlates with significantly lower reports of vagueness when compared to unintentional mind wandering (Seli et al., 2017: 152). This supports our claim that some purported instances of intentional mind wandering can be re-described as focused daydreaming.

This does not imply, however, that every purported instance of intentional mind wandering is actually an instance of focused daydreaming. We think that many purported instances of intentional mind wandering are actually instances of motivated task switching. Consider, for example, a case where somebody is taking a road trip through central Idaho. They currently find themselves driving down a long, flat stretch of highway. This individual might stop consciously attending to the road and begin thinking about a paper she's writing or a talk that she's giving in a few weeks. Again, this appears to be a case of intentional mind wandering, but an interpretation compatible with the Standard View is available. 
Repeated performance of some activity makes that activity overlearned. Here, we are thinking of tasks as activities that executive resources (such as attention and cognitive control) supervise (Mole, 2011: 51). Christopher Mole (2011: 62) explains:

It is typically harder to give sustained attention to familiar, wellunderstood tasks than it is to give attention to tasks that are poorly understood. The thought... is that giving one's attention to a wellunderstood task involves marshalling a large set of resources, just because the task is so well-understood.

When there are too many available resources, executing an activity as a task (where this implies guided, attentive performance) can hurt performance. Consider, for instance, that tying one's shoes or walking up stairs is more difficult when one focuses on every component of the activity. Thus, there is some incentive to switch tasks when one is performing an overlearned activity.

In other cases, there is no reason to engage in focused thinking when performing an overlearned activity. When an activity is easy or there is a history of successfully performing the activity without difficult thinking, there is some incentive to shift one's focus to something else that might benefit from focused, engaged thinking (see Boureau, Sokol-Hessner, \& Daw, 2015; Kool, Gershman, \& Cushman, 2017). This might be what's happening in the driving case. The individual has a long history of driving successfully without thinking very hard about driving, the roads are flat, the traffic is thin, and visibility is good. So, there is some motivation to shift the focus of one's thinking to something else — such as a paper or presentation — that could use some focused attention.

More formally, we suggest that an agent (S) can focus on one of several different task sets $\left(\mathrm{T}_{1}, \mathrm{~T}_{2}, \ldots, \mathrm{T}_{\mathrm{n}}\right)$ at any given time (e.g., focus on driving, focus on the paper, focus 
on the presentation, etc.). The marginal utility of focusing on a given task set decreases monotonically over time. So, $\mathrm{S}$ might be focused on $\mathrm{T}_{1}$, though the cost of focusing on $\mathrm{T}_{1}$ outweighs the benefits of focusing on $\mathrm{T}_{1}$ relative to the associated costs and benefits of focusing on either $T_{2}$ or $T_{n}$. At this point, $S$ might decide to shift focus from $T_{1}$ to $T_{2}$ (e.g., shifting focus from driving to paper writing). This might appear to be a case of intentional mind wandering, as there is a decision to shift focus away from an ongoing activity. But this is just an instance of motivated task switching, where $\mathrm{S}$ swaps out $\mathrm{T}_{1}$ for $\mathrm{T}_{2}$ or $\mathrm{T}_{\mathrm{n}}$. In this situation, the motivated task switching is not mind wandering, because the individual is engaged in focused, goal-directed cognition (thinking about the paper or the presentation).

This proposal is consistent with other theoretical explanations of intentional mind wandering as a kind of strategic cognition. For example, Seli and colleagues explain that intentional mind wandering manifests a strategic allocation of psychological resources because such allocations enable more locally beneficial forms of thinking (Seli et al., 2018-a). Another similar proposal is the current concerns hypothesis, which claims that mind wandering occurs when focusing on internal goals and desires has a higher incentive value than attending to the external environment (Klinger, 1987; Klinger, Barta, \& Maxeiner, 1980; Klinger, Gregoire, \& Barta, 1973). The key distinction that we make here is that when a person intends to think about their internal or long-term goals, she is switching tasks from the experimental paradigm to an internally-oriented task set. One difference, then, between mind wandering and task switching is this. When an agent task switches from $T_{1}$ to $T_{2}$, the agent reorganizes the structure of her activity such that she is primarily disposed to be attentive toward different kinds of consideration that are more 
relevant to $\mathrm{T}_{2}$ and correspondingly less disposed to be attentive toward considerations that are relevant to $T_{1}$. When an agent mind wanders from $T_{1}$ to $T_{2}$, the agent is still primarily disposed to be attentive to considerations relevant to $T_{1}$ and will feel distracted when she becomes aware of her mind wandering. ${ }^{10}$

We are not claiming that every purported instance of intentional mind wandering is either an instance of focused daydreaming or motivated task switching. The aforementioned examples suggest how one might interpret apparent cases of intentional mind wandering as instances of other well-defined psychological categories. This is the heart of the eliminativist approach to intentional mind wandering that the Standard View must adopt.

One problem with rejecting intentional mind wandering is that people report intentional mind wandering when prompted to distinguish between goal-directed thinking, unintentional, and intentional mind wandering (e.g., Seli, Carriere, \& Smilek, 2015; Seli, Risko, \& Smilek, 2016; Seli et al., 2017; Seli et al., 2018-b). Similar findings come from studies of mind wandering in daily life, where people self-report letting their minds wander on purpose (Kane et al., 2007). Additionally, there is evidence from experimental philosophy that suggests the folk concept of mind wandering recognizes something like intentional mind wandering (Irving et al., Manuscript). The Standard View, then, cannot just re-interpret purported instances of intentional mind wandering. The view must also include some kind of error theory to explain away people's judgments about their own mind wandering. We do not have enough space to develop a

\footnotetext{
${ }^{10}$ Our explanation of the difference between task-switching and mind wandering relies heavily on the notion of guidance developed in Irving (2016). We think that there is more to say about the relative utility functions that describe the values of different task sets and how those figure into an explanation of mind wandering on the Standard View. That, however, is an issue that requires separate treatment.
} 
fully adequate error theory. We simply sketch an outline of one such theory, noting that the Standard View owes an error theory about why people are systematically mistaken about the existence of intentional mind wandering.

In one of the earliest daily-life studies of mind wandering, Kane and colleagues (2007) found that people frequently reported letting their minds wander on purpose. Consistent with this, people did not report feeling surprised by their mind wandering (Kane et al., 2007: 618; see also Kane et al., 2017). One issue with these results is that people might exhibit a social desirability bias in their responses (see Weinstein, 2018). For example, people might have a desirability to manifest control to others, which would explain why they respond that their mind wandering is not surprising and that their mind wandering happens on purpose. Additionally, as we argue below, judgments of intentional mind wandering may reflect post hoc inferences anchored to properties of one's experience that are not constitutive of mind wandering.

One factor that might explain reports of intentional mind wandering in laboratory settings is that participants have a mistaken conception of mind wandering. In an experimental context, participants are told to perform the task provided by the experimenter. Thus, when participants are asked whether their minds were wandering, they might categorize any thoughts unrelated to the researcher-imposed task as mind wandering. However, it might be the case that participants chose to switch from the researcher-imposed task to an internally-oriented task (perhaps due to boredom or mental fatigue or some other reason). In this case, participants report intentional mind wandering because they chose to think about something unrelated to the experimental task. Notice, however, that this might not be a case of mind wandering, as it is possible for the 
participant to engage in focused thinking about something unrelated to the experimental task.

This explanation is consistent with our picture of motivated task switching presented earlier. Experimental tasks are often boring, and there is little incentive for participants to fully focus on the task. Because there are likely other things that are more rewarding for the individual to think about, there is a higher likelihood that participants will choose to shift the focus of attention to something else. We suggest that participants are also likely to label as 'intentional mind wandering' any thoughts that are unrelated to the researcher-imposed task even when those thoughts are guided toward a single task or topic. This mistake might be one source of self-reported intentional mind wandering.

As we noted before, partisans of the Standard View need to have something to say about the apparent existence of intentional mind wandering. We have gestured at some points that can be made to start explaining away the evidence that seems to weigh in favor of intentional mind wandering.

\section{Conclusion}

Our argument generates a surprising result: either the Standard View is mistaken or there is no such thing as intentional mind wandering. We think that the argument has implications for many of the major theories of mind wandering — including Dynamic and Family Resemblance views - but the biggest implication is for the Standard View. The Standard View is overwhelmingly popular in cognitive science. We have suggested that researchers either drop the Standard View or begin working on ways to explain away the evidence for intentional mind wandering. Either way, as philosophical interest in mind 
wandering increases, this represents an exciting frontier in constructing a theory of mind wandering. 


\section{REFERENCES}

Amaya, S. 2018. "Two kinds of intentions: a new defense of the Simple View," Philosophical Studies $175: 7,1767-1786$.

Andrews-Hanna, J.R., Irving, Z.C., Fox, K.C.R., Spreng, N., and Christoff, K. (2018). "The Neuroscience of Spontaneous Thought: An Evolving, Interdisciplinary Field," in K. Fox and K. Christoff (eds.), Oxford Handbook of Spontaneous Thought and Creativity (Oxford: Oxford University Press).

Aune, B. 1966. “Intention and foresight,” Journal of Philosophy 63:20, 652-54.

Baird, B., Smallwood, J., and Schooler, J.W. 2011. "Back to the future: Autobiographical planning and the functionality of mind-wandering," Consciousness and Cognition 20, 1604-1611.

Boureau, Y.-L., Sokol-Hessner, P., Daw, N.D. 2015. "Deciding How to Decide: Self-Control and MetaDecision Making," Trends in Cognitive Sciences 19:11, 700-10.

Bratman, M.E. 1984. “Two Faces of Intention,” Philosophical Review 93:3, 375-405.

Bratman, M.E. 1987. Intention, Plans, and Practical Reason (Cambridge, MA: Harvard University Press).

Butler, R.J. 1978. “Report on Analysis 'Problem' no. 16,” Analysis 38:3, 113-14.

Christoff, K., Irving, Z., Fox, K., Spreng, R.N., Andrews-Hanna, J. 2016. "Mind-wandering as spontaneous thought: a dynamic framework," Nature Reviews Neuroscience 17, 718-31.

Dorsch, F. 2015. "Focused Daydreaming and Mind-Wandering," Review of Philosophy and Psychology 6:4, 791-813.

Giambra, L. 1995. "A laboratory method for investigating influences on switching attention to taskunrelated imagery and thought," Consciousness and Cognition 4:1, 1-21.

Herdova, M. 2018. "Trigger Warning: No proximal intentions required for intentional action," Philosophical Explorations 21:3, 364-383.

Irving, Z. 2016. "Mind-wandering is unguided attention: accounting for the "purposeful wanderer," Philosophical Studies 173, 547-571.

Irving, Z. Manuscript. "Drifting and Directed Minds," Available on PhilPapers:

https://philarchive.org/rec/IRVDAD?all_versions=1.

Irving, Z. and Thompson, E. 2018. "The Philosophy of Mind-Wandering," in K. Fox and K. Christoff (eds.) Oxford Handbook of Spontaneous Thought (Oxford: Oxford University Press).

Irving, Z., Glasser, A., Gopnik, A., and Sripada, C. Manuscript. "What does 'Mind-Wandering' Mean to the Folk? An Empirical Investigation,” Available on PsyArxiv: psyarxiv.com/su2qj/.

Kane, M. J., Brown, L. H., McVay, J. C., Silvia, P. J., Myin-Germeys, I., \& Kwapil, T. R. (2007). For whom the mind wanders, and when: An experience-sampling study of working memory and executive control in daily life. Psychological science, 18(7), 614-621. 
Kane, M. J., Gross, G. M., Chun, C. A., Smeekens, B. A., Meier, M. E., Silvia, P. J., \& Kwapil, T. R. (2018). For whom the mind wanders, and when, varies across laboratory and daily-life settings.

Psychological science, 28(9), 1271-1289.

Klinger, E. (1987). Current concerns and disengagement from incentives. In Motivation, intention, and volition (pp. 337-347). Springer, Berlin, Heidelberg.

Klinger, E., Barta, S. G., \& Maxeiner, M. E. (1980). Motivational correlates of thought content frequency and commitment. Journal of Personality and Social Psychology, 39(6), 1222.

Klinger, E., Gregoire, K. C., \& Barta, S. G. (1973). Physiological correlates of mental activity: Eye movements, alpha, and heart rate during imagining, suppression, concentration, search, and choice. Psychophysiology, 10(5), 471-477.

Kool, W., Gershman, S.J., and Cushman, F.A. 2017. "Cost-Benefit Arbitration Between Multiple Reinforcement-Learning Systems," Psychological Science 28:9, 1321-33.

Kraemer, E.R. 1978. "Intentional Action, Chance and Control," Analysis 38:3, 116-17.

Martel, A., Arvaneh, M., Robertson, I., Smallwood, J., and Dockree, P. In Preparation. "Distinct neural markers for intentional and unintentional task unrelated thought." Available on bioRxiv doi: http://dx.doi.org/10.1101/705061.

Mele, A.R. 2009. "Mental Action: A Case Study," in L. O'Brien and M. Soteriou (eds.), Mental Actions (Oxford: Oxford University Press), 17-37.

Mele, A.R. Forthcoming. “On snubbing proximal intentions," Philosophical Studies.

Metzinger, T. 2013. "The myth of cognitive agency: subpersonal thinking as a cyclically recurring loss of mental autonomy," Frontiers in Psychology 4. doi: 10.3389/fpsyg.2013.00931.

Mills, C., Raffaelli, Q., Irving, Z., Stan, D., and Christoff, K. 2018. "Is an off-task mind a freely-moving mind? Examining the relationship between different dimensions of thought," Consciousness and Cognition $58,20-33$.

Mole, C. 2011. Attention is Cognitive Unison (Oxford: Oxford University Press).

Ross, D. 1978. “He Loads the Gun, Not the Dice,” Analysis 38:3, 114-15.

Seli, P., Carriere, J., and Smilek, D. 2015. "Not all mind wandering is created equal: dissociating deliberate from spontaneous mind wandering," Psychological Research 79, 750-758.

Seli, P., Risko, E., Smilek, D., Schacter, D. 2016. "Mind-Wandering With and Without Intention,” Trends in Cognitive Sciences 20:8, 605-617.

Seli, P., Risko, E., and Smilek, D. 2016. "On the Necessity of Distinguishing Between Unintentional and Intentional Mind Wandering," Psychological Science 27:5, 685-91.

Seli, P., Wammes, J. D., Risko, E. F., \& Smilek, D. 2016. On the relation between motivation and retention in educational contexts: The role of intentional and unintentional mind wandering. Psychonomic bulletin \& review, 23(4), 1280-1287.

Seli, P., Ralph, B., Konishi, M., Smilek, D., and Schacter, D. 2017. "What did you have in mind?

Examining the content of intentional and unintentional types of mind wandering," Consciousness and

Cognition 51, 149-56. 
Seli, P., Carriere, J., Wammes, J., Risko, E., Schacter, D., and Smilek, D. 2018-a. “On the Clock: Evidence for the Rapid and Strategic Modulation of Mind Wandering," Psychological Science 29:8, 1247-56.

Seli, P., Kane, M., Smallwood, J., Schacter, D., Maillet, D., Schooler, J., and Smilek, D. 2018-b. "Mindwandering as a natural kind: A family-resemblances view," Trends in Cognitive Sciences 22:6, 479-90.

Smallwood, J. and Schooler, J. 2006. “The restless mind,” Psychological Bulletin 132:6, 946-58.

Smallwood, J. and Schooler, J. 2015. "The Science of Mind Wandering: Empirically Navigating the Stream of Consciousness," Annual Review of Psychology 66, 487-518.

Sripada, C. 2018. “An Exploration/Exploitation Tradeoff Between Mind Wandering and Goal-Directed Thinking," In Christoff, K. and Fox, K. (eds.) The Oxford Handbook on Spontaneous Thought (Oxford: Oxford University Press).

Weinstein, Y. 2018. "Mind-wandering, how do I measure thee with probes? Let me count the ways," Behavioral Research 50, 642-61.

Yaffe, G. and Vargas, M. 2014. "Introduction,” In Yaffe, G. and Vargas, M. (eds.) Rational and Social Agency: The Philosophy of Michael Bratman (Oxford: Oxford University Press), 1-11. 